\title{
Cortical activation patterns herald successful dream recall after NREM and REM sleep
}

\author{
Sarah Laxhmi Chellappa a,b ${ }^{\mathrm{a}}$, Sylvia Frey ${ }^{\mathrm{a}}$, Vera Knoblauch ${ }^{\mathrm{a}}$, Christian Cajochen ${ }^{\mathrm{a}, *}$ \\ a Centre for Chronobiology, Psychiatric Hospital of the University of Basel, Wilhelm, Kleinstrasse 27, CH-4025 Basel, Switzerland \\ ${ }^{\mathrm{b}}$ The CAPES Foundation/Ministry of Education of Brazil, Caixa Postal 365, CEP 70359 970, Brasilia-DF, Brazil
}

\section{A R T I C L E I N F O}

\section{Article history:}

Received 22 July 2010

Accepted 10 March 2011

Available online 17 March 2011

\section{Keywords:}

Dream recall

EEG spectral analysis

Cortical activity

Emotional memory

Constant routine

Sleep-wake cycle

\begin{abstract}
A B S T R A C T
Dreaming pertains to both REM and NREM sleep. However, frequency and regional specific differences in EEG activity remains controversial. We investigated NREM and REM sleep EEG power density associated with and without dream recall in 17 young subjects during a 40-h multiple nap protocol under constant routine conditions. NREM sleep was associated with lower EEG power density for dream recall in the delta range, particularly in frontal derivations, and in the spindle range in centro-parietal derivations. REM sleep was associated with low frontal alpha activity and with high alpha and beta activity in occipital derivations. Our data indicate that specific EEG frequency- and topography changes underlie differences between dream recall and no recall after both NREM and REM sleep awakening. This dual NREM-REM sleep modulation holds strong implications for the mechanistic understanding of this complex ongoing cognitive process.
\end{abstract}

(C) 2011 Elsevier B.V. All rights reserved.

\section{Introduction}

Dreaming is a universal human experience which offers a unique view of consciousness during sleep, although the "whys" and "hows" remain controversial. A critical issue of debate concerns whether dream mentation from REM and NREM sleep can be explained by a one-generator dream model or two-generator dream model. One-generator models stem from a cognitive perspective whereby physiological activity is related to dreaming from a general perspective, with cortical activation being related to the length or complexity of dream reports (Cicogna \& Bosinelli, 2001; Foulkes, 1993). In contrast, the most representative neurocognitive two-generator model (Hobson et al., 2000) proposes dream mentation as a direct function of different physiological profiles characterizing REM and NREM sleep. REM sleep high cholinergic and low aminergic neuromodulation determines dreamlike features (i.e., emotionality, bizarreness), whereas intermediate levels of cholinergic and aminergic NREM sleep neuromodulation underlies less dream mentation (Hobson et al., 2000). If this holds true, cognition occurs throughout sleep, although dream recall differs

\footnotetext{
* Corresponding author at: Centre for Chronobiology, Psychiatric Hospital of the University of Basel, Wilhelm Kleinstrasse 27, CH-4025 Basel, Switzerland. Tel.: +41 613255476; fax: +41613255577.

E-mail addresses: Sarah.Chellappa@upkbs.ch (S.L. Chellappa), Silvia.Frey@upkbs.ch (S. Frey), veraknoblauch@hotmail.com (V. Knoblauch), Christian.Cajochen@upkbs.ch (C. Cajochen).
}

due to differential activity patterns (Antrobus, 1983; Cavallero et al., 1990; Foulkes, 1993; Rosenlicht et al., 1994). Considering that both one- and two-generator models are supported by some empirical evidence (Nielsen, 2000), the psychophysiological correlates of dream recall-sleep EEG activity as a reflection of specific anatomical pathways - may help to underpin this ongoing cognitive process. Evidence suggests that dream recall relates to increased activity in REM sleep (Armitage et al., 1989), reduced alpha activity in NREM stage 2 and REM sleep (Esposito et al., 2004), and in theta ranges (Lehmann et al., 1981). This supports the idea of different neural states triggering changes in dream recall. However, strikingly few studies have investigated frequency- and topography-specific EEG characteristics of dream recall from REM and NREM sleep, and all with limited EEG montages (Armitage et al., 1989; Esposito et al., 2004).

Here we quantified NREM and REM sleep EEG activity prior to awakening in young volunteers with and without subjectively rated amount of dream recall, under stringent controlled laboratory conditions, during a 40-h multiple nap protocol. Our two main predictions were as follows:

1. Dream recall and no recall differ in a frequency-specific manner, such that alpha and theta activity will be reduced during dream recall;

2. Dream recall and no recall differ in a very topographic-specific manner, although the direction of these differences cannot be predicted. 


\section{Methods}

\subsection{Study participants}

Study volunteers were recruited through advertisements at different Swiss universities. Only candidates with Pittsburgh sleep quality index (PSQI) score $<5$ (Buysse et al., 1989) and intermediate chronotype ratings (>14 and $<21$ ) on the morning-evening M/E questionnaire (Torsvall and Åkerstedt, 1980) were enrolled. All potential participants were questioned about sleep quality, life habits and health state. Exclusion criteria were smoking, medication or drug consumption, shift work within the last 3 months, and transmeridian flights during the month prior to study. Each volunteer underwent medical exam and polysomnographically recorded adaptation night to exclude sleep disorders. Inclusion criteria were sleep efficiency $>80 \%$, $<10$ periodic leg movements/hour and an apnea-hypopnea index $<10$. Only participants without medication (with the exception of 4 young women using oral contraceptives) were included in the study. Young females started the study on day 1-5 after menses onset during the follicular phase of their menstrual cycle. Seventeen healthy young ( 9 women, 8 men, age range $20-31$ years) were included in the study. All participants gave written informed consent. The study protocol, screening questionnaires and consent form were approved by the local ethics committee and conformed to the Declaration of Helsinki.

\subsection{Study design}

One week prior to the study (baseline week), the participants were requested to abstain from excessive caffeine and alcohol (one caffeine-containing beverage per day at most and $<5$ alcoholic beverages per week). They were instructed to keep a regular sleep-wake schedule during baseline week at home (bedtimes and wake times within \pm 30 min of self-selected target time between 22:00 h and 02:00 h) prior to admission to the laboratory. Compliance was verified by sleep logs and ambulatory activity measurements (wrist activity monitor, Cambridge Neurotechnology Ltd, UK). The timing of the sleep-wake schedule during the protocol was adjusted to habitual individual bedtimes. For each participant, habitual bedtime was calculated by centring 8 -h sleep episodes during baseline week at their midpoint.

The "in-lab" part of the study comprised 2 baseline sleep episodes in the chronobiology laboratory, followed by a 40-h multiple nap protocol, with 10 alternating sleep-wake cycles of 75/150 min duration each and one recovery sleep episode (Fig. 1). Baseline and recovery nights were scheduled at individual habitual bedtimes. Polysomnography recordings and constant posture started in the afternoon after the first baseline night. Thereafter, participants remained under constant routine conditions (constant dim light levels < 8 lux during scheduled wakefulness, semi-recumbent posture in bed, food and liquid intake at regular intervals, no

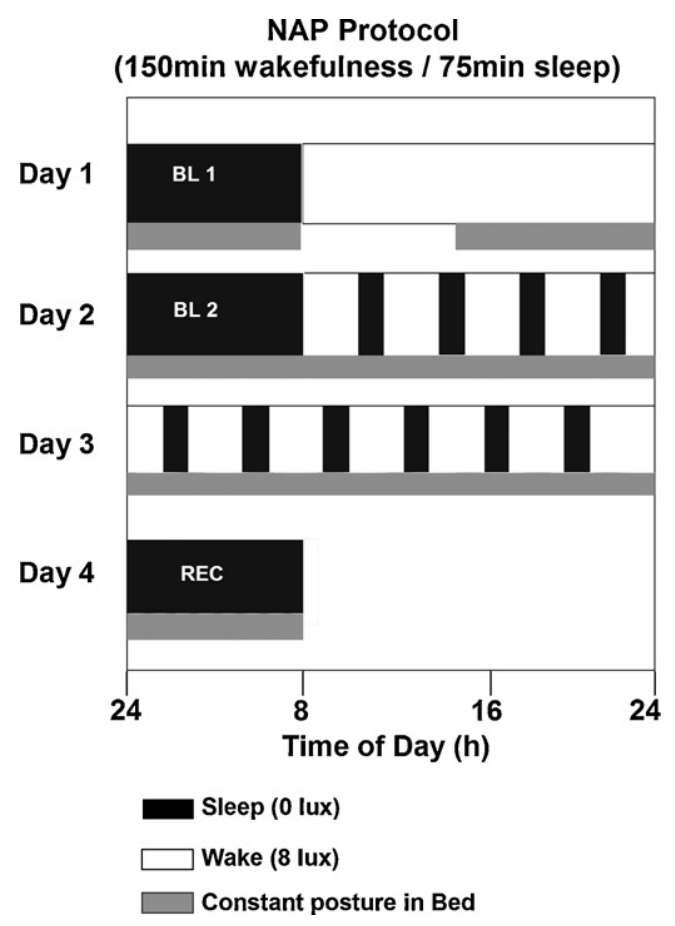

Fig. 1. Overview of the 4-day "in-lab" part of the study. Black bars (0 lux) indicate sleep and white bars wakefulness ( $<8$ lux). Gray bars indicate controlled posture (semi-recumbent during wakefulness and supine during sleep). BL: baseline night, REC: recovery night (modified from Münch et al., 2005). Herein, data comprise 40-h between BL 2 and recovery night (multiple-nap protocol). external time cues). During scheduled sleep episodes, subjects stayed in recumbent position and lights were off ( 0 lux).

\subsection{Polysomnographic measures}

Sleep was polysomnographically recorded with the VITAPORT ambulatory system (Vitaport-3 digital recorder, TEMEC Instruments B.V., Kerkrade, the Netherlands). Twelve EEGs channels, 2 electroculograms, a submental electromyogram and an electrocardiogram were recorded. All EEG signals were filtered at $30 \mathrm{~Hz}$ (fourth-order Bessel-type antialiasing low-pass filter, total $24 \mathrm{~dB} / \mathrm{Oct}$ ), and a time constant of $1.0 \mathrm{~s}$ was used prior to online digitization (range $610 \mu \mathrm{V}, 12$ bit AD converter, $0.15 \mu \mathrm{V} / \mathrm{bit}$; storage sampling rate at $128 \mathrm{~Hz}$ ). The raw signals were stored online on a Flash RAM Card (Viking, USA) and downloaded offline to a PC hard drive. Sleep stages were visually scored per 20-s epochs (Vitaport Paperless Sleep Scoring Software) according to standard criteria (Rechtschaffen and Kales, 1968).

EEG artefacts were detected by an automated artefact algorithm (CASA, 2000 PhyVision B.V., Gemert, Netherlands). Spectral analysis was conducted using a Fast Fourier transformation (FFT; $10 \%$ cosine 4 -s window) which yielded a $0.25 \mathrm{~Hz}$ bin resolution. NREM sleep (stages) and REM sleep were expressed as the percentage of total sleep time per nap before averaging over subjects. EEG power spectra were calculated during REM sleep and NREM sleep in the frequency range from 0 to $20 \mathrm{~Hz}$. Finally, artefact free 4-s epochs were averaged over 20-s epochs. Here, we report log-transformed EEG data derived from 12 topographical derivations (F3, F4, Fz, C3 $\mathrm{C} 4, \mathrm{Cz}, \mathrm{P} 3, \mathrm{P} 4, \mathrm{Pz}, \mathrm{O} 1, \mathrm{O} 2$, and $\mathrm{Oz})$ referenced against linked mastoids $(\mathrm{A} 1, \mathrm{~A} 2)$ in the range of $0.5-20 \mathrm{~Hz}$ on log-transformed data.

A nap trial that contained only REM sleep in the last 15 min of a scheduled 75min nap was defined as a REM nap and a nap trial with NREM sleep (stags 1-4) in the last 15 min was defined as a NREM nap (Chellappa et al., 2009). "Wakefulness naps" were defined as nap trials not containing either NREM or REM sleep stages and were excluded from further analyses.

The amount of REM and/or NREM sleep stages during the 15 min prior to the selected last 15 min of a given nap was calculated and the ratio of NREM/REM sleep in both of these two 15-min intervals was compared to illustrate if these ratios remain similar or not in both intervals across the 40-h nap protocol (see supplementary Fig. $1)$.

\subsection{Dream recall}

Dream recall was assessed immediately at the end of each nap trial (10 naps in total) with the Sleep Mentation Questionnaire, which addresses numerous characteristics of dream recall, such as number of dreams, emotionality, vividness, pleasantness, hostility, and colourfulness, on a likert-point scale, whereby 1 : greatly, 2: fairly, 3: little, and 4: not at all (Chellappa et al., 2009). Because the 40-h protoco assesses dream recall at a very high sampling frequency (in addition to collecting physiological variables and carrying out performance tests), we did not access dream content, as this could have seriously influenced and, therefore, biased dream reports at successive nap trials. Instead we selected the previously validated, readily comprehensible, sleep mentation questionnaire (Chellappa et al., 2009), whereby subjectively rated amount of dream experiences could be quantified as either dream or no dream recall. For this categorized classification of dream recall, only the first question [Q1. "How much did you dream?" (1: greatly, 2: fairly, 3: little, and 4: not at all)] was considered for all the analysis of our dataset. Participants were considered to have had dream recall if their response to Q1 was not 4.

\subsection{Salivary melatonin and classification of biological day and night}

Saliva collections were scheduled during wakefulness every $30 \mathrm{~min}$. A direct double-antibody radioimmunoassay was utilized for the melatonin assay (validated by gas chromatography-mass spectroscopy with an analytical least detectable dose of $0.65 \mathrm{pm} / \mathrm{mL}$; Bühlmann Laboratory, Schönenbuch, Switzerland) (Weber et al. 1997). For mean melatonin levels, values of all samples between the upward- and downward-mean crossing points were averaged per subject. The mean melatonin concentration was calculated for each subject. A nap was classified as a night nap (biological night) if the melatonin concentration of the last saliva sample prior to the nap was above the individual mean; otherwise, it was classified as a day nap (biological day) (Knoblauch et al., 2005; Münch et al., 2005). This classification was carried out since throughout the 10 scheduled naps, not every scheduled nap had comparable levels of dream recall. Naps were classified into two categories of biological day or night naps-given that by comprising more naps, the comparison of dream recall between these two nap categories would be viable. On average, there were $6.9 \pm 0.4$ day naps and $3.1 \pm 0.2$ night naps per subject.

\subsection{Statistical analysis}

For all analysis, the statistical packages SAS (SAS Institute Inc., Cary, NC, USA Version 6.12) and Statistica (Stat-Soft Inc., 2000-2004, STATISTICA for Windows, Tulsa, OK, USA) were utilized. The ratio of NREM/REM sleep of the last $15 \mathrm{~min}$ of a scheduled nap and the $15 \mathrm{~min}$ prior to the abovementioned time interval of the same nap were compared with one-way ANOVA (Factor: 'time'). For recall-no recall comparisons during NREM and REM sleep, the mixed-model analyses of vari- 
ance for repeated measures, r-ANOVA (PROC MIXED), was performed with factors 'dream recall' (recall or no recall) and 'derivation' (frontal = F3, F4 and Fz; central = C3, $\mathrm{C} 4$ and $\mathrm{Cz}$; parietal=P3, P4 and Pz; occipital=01, 02 and $\mathrm{Oz}$, thus 4 levels of the repeated measures factor 'derivation'). Alpha adjustment for multiple comparisons was applied in PROC MIXED using Tukey-Kramer test. For day-night comparisons during NREM and REM sleep (please see supplementary material), averaged EEG power density across biological daytime naps was compared with averaged values across biological night-time naps for both recall and no recall conditions. Spectral results averaged for each recall and no recall nap for each subject, as well as for the biological day/night comparisons. Three-way rANOVA (PROC GLM) with the factors dream recall (recall or no recall), 'biological condition' (biological day and night) and 'derivation' ( frontal = F3, F4 and Fz; central = C3, C4 and Cz; parietal = P3, P4 and Pz; occipital = 01, $\mathrm{O} 2$ and $\mathrm{Oz}$ ) was performed. Alpha adjustment for multiple comparisons was applied in PROC MIXED using Tukey-Kramer test. For factor 'derivation', the corresponding three derivations (i.e. Frontal =F3, F4 and Fz) were averaged per subject, given that there were no lateralisation effects for dream recall and no recall. Gender was included as covariate in this model, in which the same factors were utilized. All $p$-values derived from rANOVAs were based on Huynh-Feldt's (H-F) corrected degrees of freedom (significance level: $p<0.05$ ).

\section{Results}

\subsection{Sleep stages during naps}

Sleep stages during the 10 scheduled naps (indexed as percentage of baseline night) indicate that, respectively, NREM sleep stage 1 comprised $21.7 \pm 8.6 \%$, stage 2 was $44.6 \pm 17.3 \%$, stage 3 was $13.5 \pm 4.1 \%$, stage 4 was $9.0 \pm 5.9 \%$ and REM sleep was $15.6 \pm 7.5 \%$ (all values mean \pm SEM, \% total sleep time - TST). Time course analysis of TST, NREM sleep, and REM sleep within the naps yielded less TST during naps 4 and 10 (respectively, time of day: Day 1, 22:00 h, and Day 2, 20:00 h) (wake maintenance zone) and more TST during naps 5 and 8 (respectively, time of day: Day 2, 02:00 h, and Day 2, 13:00 h). Less NREM sleep was elicited during nap 4 (time of day: Day 1, 22:00 h) and nap 10 (time of day: Day 2, 20:00 h), while REM sleep was significantly higher during nap 1 (time of day: Day 1 , 11:00 h), nap 6 (time of day: Day 2, 05:00 h) and nap 7 (time of day: Day 2, 09:00 h) (1-way ANOVA, factor 'time', $p<0.05)$. Time of day corresponds to mean values across subjects. For detailed information on sleep during the naps, please see (Münch et al., 2005,2007 ). Table 1 (please see supplementary material) illustrates sleep stages derived from visual scoring for dream recall and no recall, averaged across interval 5 (last $15 \mathrm{~min}$ ) of naps $1-10$. As indicated, no differences were elicited for total sleep time, sleep efficiency, NREM 1-4 sleep stages and slow-wave sleep. No recall yielded a tendency for more NREM sleep (Wilcoxon matched pair test, $p<0.1$ ), while dream recall was associated with significantly more REM sleep (Wilcoxon matched pair test, $p<0.05$ ).

\subsection{NREM sleep EEG power spectra prior to dream recall and no-dream recall}

Overall dream recall rate during NREM sleep was $57 \pm 9.6 \%$. No differences were observed for gender effects, as well as in all further analysis of this data set. A significant interaction between the factors 'derivation' (frontal, central, parietal, and occipital) and 'recall' (dream recall vs. no dream recall) was found in the delta $(1-3 \mathrm{~Hz})$ (two-way r-ANOVA on log-transformed values, $\left.F_{3,57}=7.81 ; p<0.05\right)$ and spindle $(12-15 \mathrm{~Hz})$ (two-way r-ANOVA on log-transformed values, $\left.F_{3,57}=3.65 ; p<0.05\right)$, but not in theta $(4.75-7.75 \mathrm{~Hz})$, alpha $(8-9.75 \mathrm{~Hz})$ and beta ranges $(16.5-20 \mathrm{~Hz})$. Accordingly, delta activity was attenuated prior to dream recall particularly in fronto-central brain regions $(p<0.001)$, with a concomitant spindle decrease in centro-parietal brain regions $(p=0.013)$, as compared to no recall (Fig. 2). For enhanced visual illustration, a global cortical visual plot with the entire topography is provided in Fig. 3, for the EEG power density in the delta and spindle range after dream recall and no recall, and for the EEG anterior-posterior topography gradient (EEG recall and no recall
NREM sleep EEG spectra

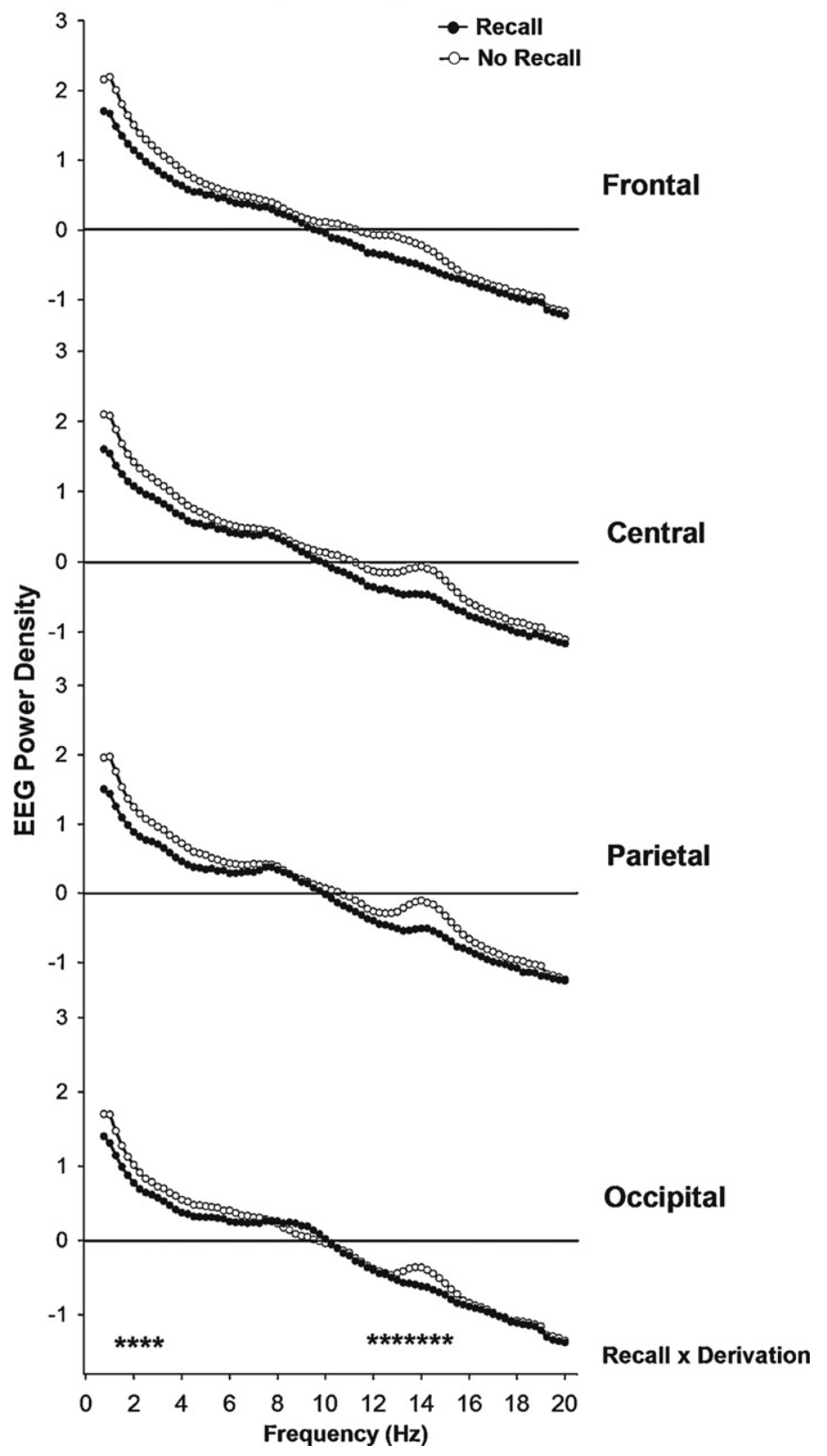

Fig. 2. Absolute NREM sleep EEG spectra (log-transformed) during recall (black circles) and no recall (white circles) $(n=17)$ in the frequency range between 0.75 and $20 \mathrm{~Hz}$ for frontal, central, parietal and occipital derivations. Stars near the abscissa indicate frequency bins with a significant interaction 'recall' $x$ 'derivation' $(p<0.05)$.

spectra expressed as relative ratio of recall/no recall values). Dream recall was related to less delta, particularly in fronto-central derivations, and spindle activity in centro-parietal derivations (two-way r-ANOVA conducted on log-transformed data, factors 'derivation' and 'recall', $\left.F_{3,54} ; p<0.05\right)$.

\subsection{REM sleep EEG power spectra prior to dream recall and no-dream recall}

Overall dream recall percentage during REM sleep was $74 \pm 13.5 \%$. A significant interaction between factors 'derivation' and 'recall' occurred for the alpha $(10-12 \mathrm{~Hz}$ ) (two-way r-ANOVA on log-transformed values, $\left.F_{3,59}=13.35 ; p<0.001\right)$ and beta $(14-19 \mathrm{~Hz})$ (two-way r-ANOVA on log-transformed values, $\left.F_{3,59}=3.99 ; p=0.03\right)$, but not for delta $(1-3.5 \mathrm{~Hz})$ and theta 

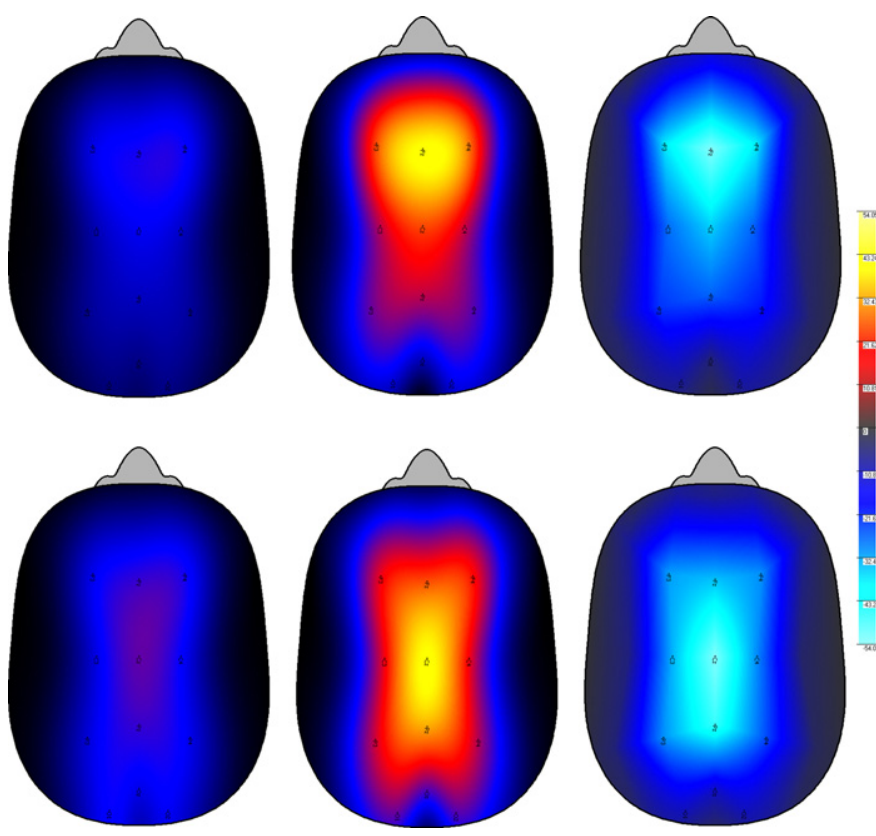

Fig. 3. Top panel illustrates the topographical NREM sleep EEG power density in delta activity $(1-3 \mathrm{~Hz})$ during dream recall and no recall. Top panels left, middle and right depict, respectively, NREM delta activity during dream recall, no recall, and the relative NREM delta activity (relative ratio of dream recall/no recall). Bottom panel illustrates the topographical NREM sleep EEG power density in spindle activity $(12-15 \mathrm{~Hz})$ during dream recall and no recall. Bottom panels left, middle and right depict, respectively, NREM spindle during dream recall, no recall, and the relative NREM spindle activity (ratio of dream recall/no recall). Scales: light blue indicates minimum EEG activity and yellow indicates maximum. (For interpretation of the references to color in this figure legend, the reader is referred to the web version of the article.)

$(4.75-7.75 \mathrm{~Hz})$ ranges. While delta and theta ranges during dream recall yielded no significant differences, alpha and beta ranges were lower in the frontal derivation $(p<0.001)$ and higher in the occipital derivation ( $p=0.013$ ) (Fig. 4). For enhanced visual illustration, a global cortical visual plot with the EEG anterior-posterior topography is provided in Fig. 5 for the frequencies (alpha and beta ranges) in which significant differences were elicited between recall and no recall. Accordingly, dream recall was related to lower alpha activity in frontal derivation and higher in occipital derivation, together with higher beta activity in the occipital derivation.

\section{Discussion}

Our data yielded distinct frequency- and topography specific spectral EEG correlates for subjectively rated amount of dream recall during NREM and REM sleep. Dream recall was preceded by lower frontal delta NREM EEG activity and lower spindle NREM EEG activity in centro-parietal brain regions, together with higher REM alpha and beta activity in the occipital cortex.

\subsection{NREM sleep is not a challenge for dream recall}

NREM dreaming challenges the concept that REM-specific physiology underlies all sleep mentation (Cavallero et al., 1992). However, it remains controversial if NREM dreams are the repercussion of "covert" REM sleep (Nielsen, 2000). For instance, dreaming scores elicited for NREM dream recall can be distributed sinusoidally across the 24-h day, with the NREM dreaming curve parallel to the REM sleep curve, as indexed by time-in-stage prior to awakening, which suggests that the dreaming output from REM and NREM sleep may be influenced by the same underlying circadian oscillator (Suzuki et al., 2004). In our study, the percentage

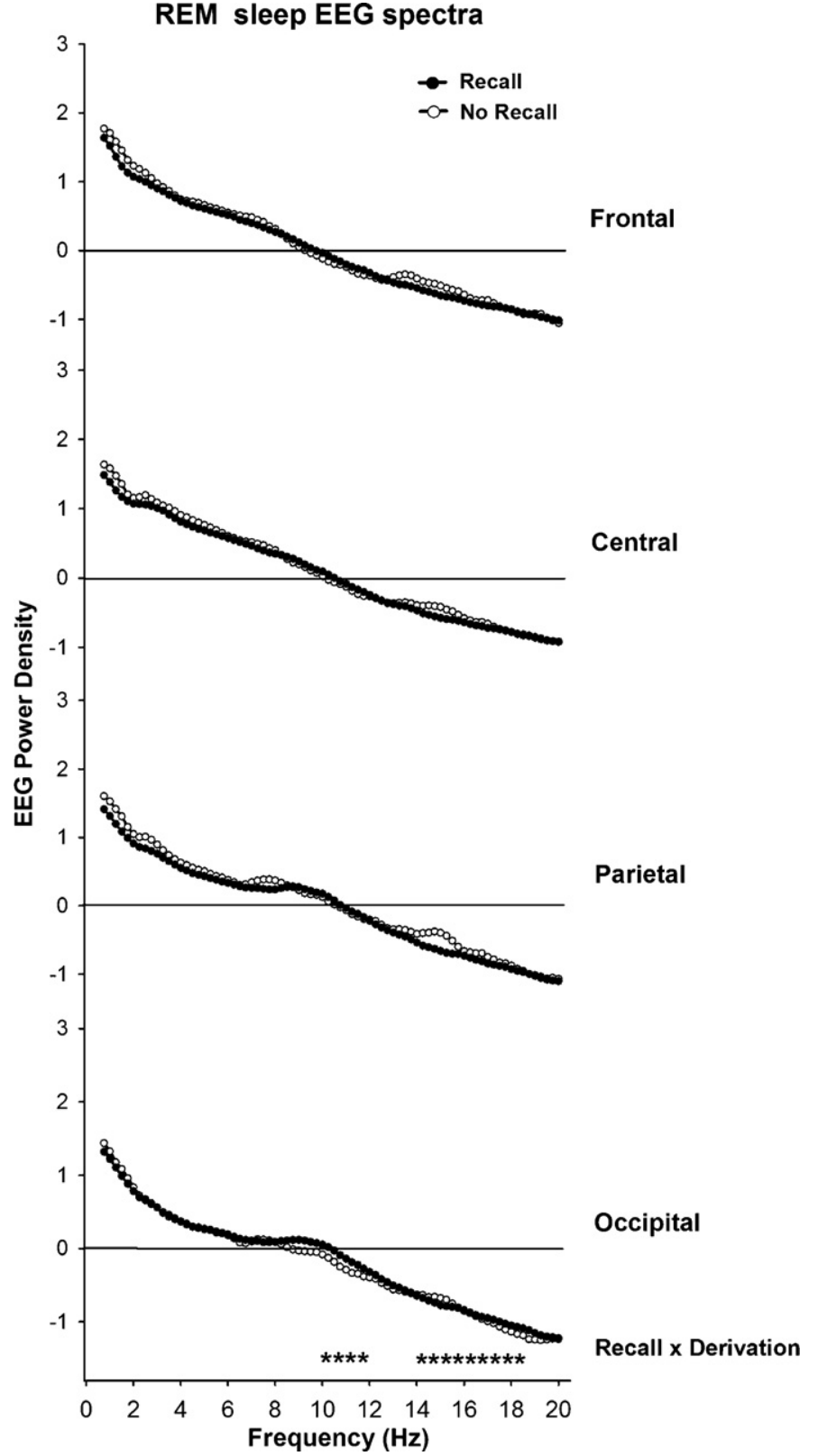

Fig. 4. Absolute REM sleep EEG spectra (log-transformed) during recall (black circles) and no recall (white circles) in the frequency range between 0.75 and $20 \mathrm{~Hz}$ for frontal, central, parietal and occipital derivations. Stars near the abscissa indicate the frequency bins with a significant interaction 'recall' $x$ 'derivation' $(p<0.05)$.

of NREM sleep and REM sleep during 15 minutes prior to awakening and 15 minutes prior to this time window had similar levels (supplemental Fig. 1), although this does not rule out that NREM sleep preceding dream recall may contain REM-like signs, such as lower EMG and increased rapid eye movements. Furthermore, our EEG findings for dream recall in NREM naps, such as decreased delta and spindle power, seem consistent with the idea that dreaming is more likely if sleep contains fewer of the classical defining signs of NREM sleep (delta waves, spindles). In other words, dreaming in NREM sleep could be more likely if this sleep stage is relatively more 'REM-like' (Nielsen, 2000) or, alternatively, that dreaming in NREM sleep waxes and wanes with variations in underlying REM sleep propensity (Nielsen, 2004).

NREM dream recall was associated with lower frontal delta and centro-parietal spindle activity. The intrinsic thalamocortical network during NREM sleep, as indexed by higher levels of 

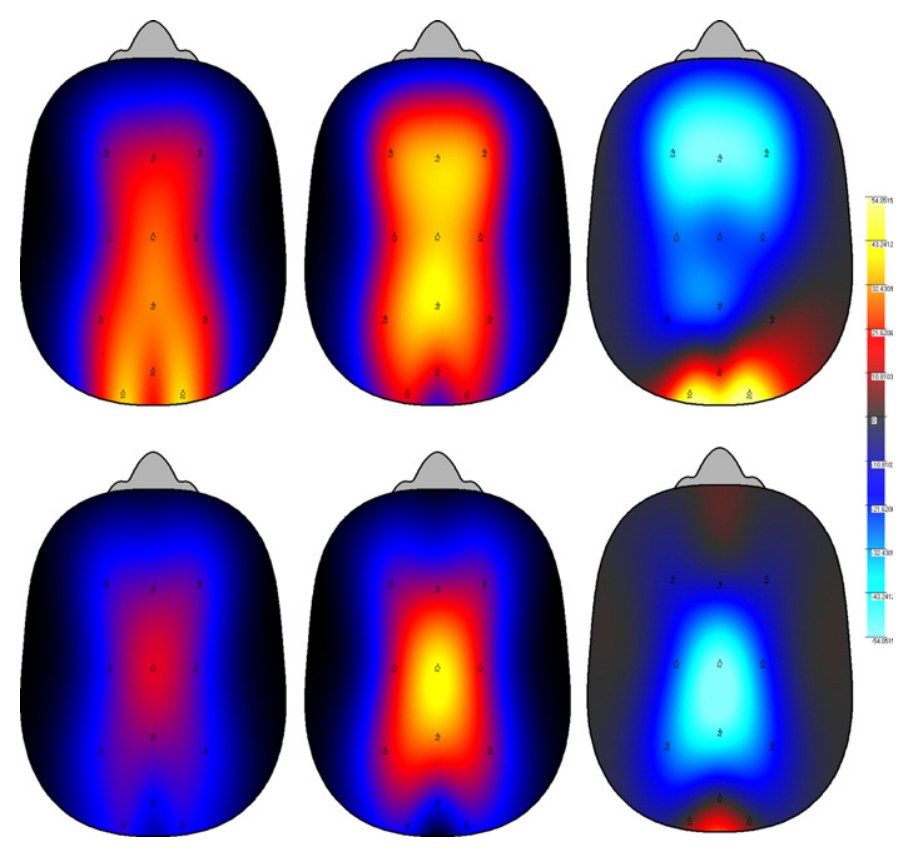

Fig. 5. Top panel illustrates the topographical REM sleep EEG power density in alpha activity $(10-12 \mathrm{~Hz})$ during dream recall and no recall. Top panels left, middle and right depict, respectively, REM alpha activity during dream recall, no recall, and the relative REM alpha activity (relative ratio of dream recall/no recall). Bottom panel illustrates the represent topographical REM sleep EEG power density in beta activity $(14-19 \mathrm{~Hz})$ during dream recall and no recall. Bottom panels left, middle and right depict, respectively, REM beta during dream recall, no recall, and the relative REM beta activity (ratio of dream recall/no recall). Scales: light blue indicates minimum EEG activity and yellow indicates maximum. (For interpretation of the references to color in this figure legend, the reader is referred to the web version of the article.)

sleep spindles and delta waves, can strongly interfere with ongoing mental activity (Steriade et al., 1993). At the transition from wakefulness to sleep, the neuronal membrane potential in the cortex and thalamus (relay station for most sensory signals to the cerebral cortex) reduces, resulting in NREM sleep oscillations - sleep spindles and slow-waves - which lead to impaired synaptic responsiveness (Timofeev et al., 2001). This neuronal network most likely explains why dream recall was dramatically reduced during delta and spindle activity.

Interestingly, we did not find differences for EEG beta activity $(16.5-20 \mathrm{~Hz})$ during NREM sleep before dream and no dream recall. Beta frequency increases in NREM stage 2, during the transition from epochs without dream mentation to those with distinct mental activity (Williamson et al., 1986), which hints to the fact that dream recall per se is likely to be mediated by high-frequency activity. This suggests that a functional stage-shift model is more appropriate than the dream recall arousal theory (Hobson et al., 2000). In other words, if the resemblance of sleep EEG patterns is nearer to those during wakefulness, there is a higher likelihood of dream recall. However, these differences usually happen in frequencies above $20 \mathrm{~Hz}$.

NREM sleep frequency-specific differences are further supported by biological day-night differences, in which dream recall was linked to lower delta and spindle activity, while no recall was related to higher spindle activity during the biological day (supplementary Fig. 2). Young subjects exhibit a well-defined circadian modulation of spindle frequency phase-locked with melatonin secretion (Knoblauch et al., 2005), with, respectively, lower and higher spindle activity during the biological night and day. One assumption is that the circadian modulation of spindle frequency facilitates consolidated night sleep and day wakefulness (Knoblauch et al., 2005). Within this framework, daytime fast spindle frequencies can represent a circadian waking signal.

\subsection{REM sleep spectral characteristics of dream recall}

Dream recall rate after REM awakening was higher in comparison to NREM sleep. During REM sleep, heightened activation of forebrain structures essential to motivation and emotion-lateral hypothalamic areas, infralimbic, prelimbic, and limbic areas (amygdaloid complex) - imposes vivid recall (Braun et al., 1997; Solms, 2000). Furthermore, motivational states with active limbic processes in REM sleep are rendered compatible with visual-spatial images in occipital and parietal cortices (Smith et al., 2004). Thus, cortical activation is biased toward REM dreaming.

We demonstrate that the offline facilitation of dream recall can be associated with lower REM alpha activity, suggesting the relationship of specific alpha activity to sleep mentation (Takeuchi et al., 2003). Esposito et al. (2004) demonstrated that dream recall was associated with lower alpha power in a non-topographic manner, which may reflect cognitive elaboration active prior to awakening. In our study, lower alpha activity was observed only in frontal derivations. REM sleep activation of sub-cortical and cortical limbic structures, which mediates emotion, can be associated with relative frontal cortex inactivation, which mediates directed thought (Braun et al., 1997). Within this context, our results might indicate that REM dreaming comprises emotion-driven cognition with possible deficient analytic thinking.

Another key finding was the association of dream recall with higher alpha and beta activity in the occipital cortex. The activation synthesis model (Hobson and McCarley, 1977) proposes PGO (ponto-geniculo-occipital) activity as a generator of the internal signal and sensory input of REM sleep, whereby the brainstem is the key initiator of dreaming, assigning the forebrain a secondary role. The forebrain then receives tonic and phasic signals from the midbrain reticular formation via thalamus, and phasic eye movement signals from the pontine reticular formation via lateral geniculate nucleus. The forebrain then compares pseudo-sensory information from the brainstem with stored sensorimotor information, resulting in dreams (Hobson and McCarley, 1977).

These frequency specific differences are further supported by the biological day-night differences, in which dream recall was related to higher alpha activity during the biological day (supplementary Fig. 3). Circadian modulation of sleep EEG is such that REM sleep propensity peak coincides with rising core body temperature during late morning hours (Dijk et al., 1997; De Gennaro and Ferrara, 2003). This can imply why dream recall maybe linked to REM alpha activity as an "electrophysiological correlate" during the biological day (Llinás and Ribary, 1993).

\subsection{Study limitations}

Our 40-h protocol assessed dream recall at a very high sampling frequency (in addition to collecting physiological variables and carrying out performance tests). For such we did not access dream content, as this could have seriously influenced and, therefore, biased dream reports at successive nap trials. For a similar reason, it may not be appropriate to speculate as to how dream content is encoded in memory during sleep and if its retrieval is facilitated after awakening (Anderson et al., 2010). Taken together, caution should be exercised when extrapolating upon how dream content and its possible EEG activity patterns may be associated, for instance, in the context of episodic memories.

\subsection{Dreaming is regulated in a very specific manner}

Recapitulating our two predictions, there are clear specificfrequency and topography dissimilarities in dream recall during NREM and REM sleep. In view of these, we suggest that (1) differences in subjective rated amount of dream recall are related 
to frequency ranges, such that higher frequencies are associated with REM sleep, while NREM dreaming can be associated with attenuated NREM sleep oscillations; (2) dreaming may be related to frontal deactivation and occipital activation. Dream variations are linked to oscillations within a specific type of rhythm, such as the REM/NREM ultradian rhythm, regulated by either single or multi-oscillatory processes (Nielsen, 2004). If single processing is correct, REM/NREM rhythm may lead to simultaneous fluctuations in dreaming, with parallel changes in dreaming as a function of ultradian (REM/NREM differences) or circadian variations (withinnight differences). If multi-processing is correct, dreaming may be regulated by separate, partially independent oscillators, as numerous physiological variables (temperature and melatonin), under circadian control. Dreaming would then have temporal morphologies seemingly out of phase with those of other measures, which can be indexed by higher recall rates in REM sleep at the end of a night sleep episode.

While our results suggest that dream mentation is NREM-REM sleep driven with frequency and topography specificity, it remains unclear if these results can exclusively fit into one of these two models. Our present findings cannot be interpreted in favour of either hypothesis of dreaming (one vs. two generators), given that we focused exclusively on dream recall and no recall, and not on measures of dream length and perceptual/emotional features of dream content. Therefore, given that the two alternative hypotheses stem from differences in the quantitative and qualitative characteristics of verbally reported dreams, our findings do not lean exclusively toward either of these models. In the broader context, this dual NREM-REM sleep modulation of dreaming may hold implications for the mechanistic understanding of this complex cognitive activity.

\section{Acknowledgments}

We thank Claudia Renz, Marie-France Dattler, and Giovanni Balestrieri for their help in data acquisition, the volunteers for participating, Dr. Antoine Viola for statistical advice, and Marcel Hofstetter for programming the visual contour plots used in this study. This work was supported by the Swiss National Science Foundation Grants (START 3100-055385.98 and 3130-054991.98) to CC and Bühlmann Laboratories, Allschwil (Switzerland) to CC.

\section{Appendix A. Supplementary data}

Supplementary data associated with this article can be found, in the online version, at doi:10.1016/j.biopsycho.2011.03.004.

\section{References}

Anderson, K., Rajagovindan, R., Ghacibeh, G., Meador, K., Ding, M., 2010. Theta oscillations mediate interaction between prefrontal cortex and medial temporal lobe in human memory. Cerebral Cortex 20 (7), 1604-1612.

Antrobus, J., 1983. REM and NREM sleep reports: comparison of word frequencies by cognitive classes. Psychophysiology 20, 562-568.

Armitage, R., Hoffmann, R., Loewy, D., Moffitt, A., 1989. Variations in period-analysed EEG asymmetry in REM and NREM sleep. Psychophysiology 26 (3), 329-336.

Braun, A.R., Balkin, T.J., Wesensten, N.J., Carson, R.E., Varga, M., Baldwin, P., Selbie, S., Belenky, G., Herscovitch, P., 1997. Regional cerebral blood flow throughout the sleep-wake cycle. An $\mathrm{H}_{2}{ }^{15} \mathrm{O}$ PET study. Brain 120, 1173-1197.

Buysse, D., Reynolds III, C., Monk, T., Berman, S., Kupfer, D., 1989. The Pittsburgh sleep quality index: a new instrument for psychiatric practice and research. Psychiatry Research 28, 193-213.
Cavallero, C., Cicogna, P., Natale, V., Occhionero, M., Zito, A., 1992. Slow wave sleep dreaming. Sleep 15 (6), 562-566.

Cavallero, C., Foulkes, D., Hollifield, M., Terry, R., 1990. Memory sources of REM and NREM dreams. Sleep 13 (5), 449-455.

Chellappa, S.L., Münch, M., Blatter, K., Knoblauch, V., Cajochen, C., 2009. Does the circadian modulation modify with age? Sleep 32 (9), 1201-1209.

Cicogna, P.C., Bosinelli, M., 2001. Consciousness during dreams. Conscious Cognition $10(1), 26-41$

De Gennaro, L., Ferrara, M., 2003. Sleep spindles: an overview. Sleep Medicine Reviews 7 (5), 423-440.

Dijk, D.J., Shanahan, T.L., Duffy, J.F., Ronda, J.M., Czeisler, C.A., 1997. Variation of electroencephalographic activity during non-rapid eye movement and rapid eye movement sleep with phase of circadian melatonin rhythm in humans. Journa of Physiology 505, 851-858.

Esposito, M.J., Nielsen, T.A., Paquette, T., 2004. Reduced alpha power associated with the recall of mentation from stage 2 and stage REM sleep. Psychophysiology 41 (2), 288-297.

Foulkes, D., 1993. Normal and abnormal REM sleep regulation: dreaming and REM sleep. Journal of Sleep Research 2 (4), 199-202.

Hobson, J.A., McCarley, R.W., 1977. The brain as a dream state generator: an activation-synthesis hypothesis of the dream process. American Journal of Psychiatry 134 (12), 1335-1348.

Hobson, J.A., Pace-Schott, E.F., Stickgold, R., 2000. Dreaming and the brain: toward a cognitive neuroscience of conscious states. Behavioral and Brain Sciences 23 (6), 793-842.

Knoblauch, V., Münch, M., Blatter, K., Martens, W.L., Schröder, C., Schnitzler-Sack, C., Wirz-Justice, A., Cajochen, C., 2005. Age-related changes in the circadian modulation of sleep-spindle frequency during nap sleep. Sleep 28 (9), 1093-1101.

Lehmann, D., Dumermuth, G., Lange, B., Meier, C., 1981. Dream recall related to EEG spectral power during REM periods. Journal of Sleep Research 10, 191-192.

Llinás, R., Ribary, U., 1993. Coherent 40-Hz oscillation characterizes dream state in humans. Proceedings of the National Academy of Sciences of the United States of America 90 (5), 2078-2081.

Münch, M., Knoblauch, V., Blatter, K., Schröder, C., Schnitzler-Sack, C., Kräuchi, K. Wirz-Justice, A., Cajochen, C., 2005. Age-related attenuation of the evening circadian arousal signal in humans. Neurobiology of Aging 26, 1307-1319.

Münch, M., Knoblauch, V., Blatter, K., Wirz-Justice, A., Cajochen, C., 2007. Is homeostatic sleep regulation under low sleep pressure modified by age? Sleep 30 (6), 781-792.

Nielsen, T.A., 2000. A review of mentation in REM and NREM sleep: “Covert” REM sleep as a possible reconciliation of two opposing models. Behavioral and Brain Sciences 23 (6), 851-866.

Nielsen, T.A., 2004. Chronobiological features of dream production. Sleep Medicine Reviews 8 (5), 403-424.

Rechtschaffen, A., Kales, A., 1968. A Manual of Standardized Terminology, Techniques and Scoring System for Sleep Stages of Human Subjects. US Dept of Health, Education and Welfare, Public Health Service, Bethesda, MD.

Rosenlicht, N., Maloney, T., Feinberg, I., 1994. Dream report length is more dependent on arousal level than prior REM duration. Brain Research Bulletin 34 (2), 99-101.

Smith, M., Antrobus, J., Gordon, E., Tucker, M., Hirota, Y., Wamsley, E.J., 2004. Motivation and affect in REM sleep and the mentation reporting process. Consciousness and Cognition 13 (3), 501-511.

Solms, M., 2000. Dreaming and REM sleep are controlled by different brain mechanisms. Behavioral and Brain Sciences 23 (6), 843-850.

Steriade, M., McCormick, D.A., Sejnowski, T.J., 1993. Thalamocortical oscillations in the sleeping and aroused brain. Science 262 (5134), 679-685.

Suzuki, H., Uchiyama, M., Tagaya, H., Ozaki, A., Kuriyama, K., Aritake, S., Shibui, K. Tan, X., Kamei, Y., Kuga, R., 2004. Dreaming during non-rapid eye movement sleep in the absence of prior rapid eye movement sleep. Sleep 27 (8), 1486-1490.

Takeuchi, T., Ogilviec, R.D., Murphy, T.I., Ferrelli, A.V., 2003. EEG activities during elicited sleep onset REM and NREM periods reflect different mechanisms of dream generation. Clinical Neurophysiology 114, 210-220.

Timofeev, I., Grenier, F., Steriade, M., 2001. Disfacilitation and active inhibition in the neocortex during the natural sleep-wake cycle: an intracellular study. Proceedings of the National Academy of Sciences of the United States of America 98 (4), 1924-1929.

Torsvall, L., Åkerstedt, T., 1980. A diurnal type scale. Construction, consistency and validation in shift work. Scandinavian Journal of Work, Environment and Health 6, 283-290.

Weber, J.M., Schwander, J.C., Unger, I., Meier, D., 1997. A direct ultrasensitive RIA for the determination of melatonin in human saliva: comparison with serum levels. Journal of Sleep Research 26, 757.

Williamson, P.C., Csima, A., Galin, H., Mamelak, M., 1986. Spectral EEG correlates of dream recall. Biological Psychiatry 21, 717-723. 